\title{
The Holomorphic Equivalence of Two Equidimensional Hartogs Domains over Bounded Symmetric Domains
}

\author{
Ting Guo and Huan Yang*
}

\begin{abstract}
This paper is concerned with the biholomorphism of two equidimensional Hartogs type domains over irreducible bounded symmetric domains $H_{\Omega}(p)$ (see $(1.1)$ ) which is a Hua construction of Cartan-Hartogs domain. We develop a new simple methods to give an sufficient and necessary condition for the two Hua domains to be biholomorphic equivalent by using the function $\mathcal{L}_{\Omega}(z, \omega)$ (see 1.2 ). Furthermore as an application, we can also give an equivalent description for the automorphism of Hua domain.
\end{abstract}

\section{Introduction}

Let $D_{1}$ and $D_{2}$ be two domains in $\mathbb{C}^{n}$. A holomorphic mapping $F: D_{1} \rightarrow D_{2}$ is said to biholomorphic if it is a one-to-one mapping and its inverse mapping $F^{-1}: D_{1} \rightarrow D_{2}$ is also holomorphic. We say that two domains $D_{1}$ and $D_{2}$ are holomorphic equivalent if there exists a biholomorphic mapping between $D_{1}$ and $D_{2}$. In particular, when $D_{1}=D_{2}$ this problem reduces to determine the automorphism group for a domain, which is an important research branch of complex analysis and has attracted a lot of attention. The study of holomorphic equivalence problem for domains in $\mathbb{C}^{n}$ is a classical problem in several complex variables and complex geometry, and the methods are relative limited. Moreover it also plays a great role in the study of the existence of Kähler Einstein metrics, proper holomorphic mapping, the existence of balanced metrics and so on (e.g., [3 6 14]). So it is interesting and of great importance to determine some holomorphic invariants for domains or more precisely to establish a kind of rigidity results about biholomorphic mappings between domains in $\mathbb{C}^{n}$.

What is encouraging is that substantial progress has been made in this field with the joint efforts of many experts. In 1968, Naruki [15] gave the sufficient and necessary condition for two equidimensional complex ellipsoids to be holomorphic equivalent. Following by this, Kodama [12] (see also [7, 13]) studied complex ellipsoid and determined

Received August 9, 2020; Accepted November 19, 2020.

Communicated by Min Ru.

2020 Mathematics Subject Classification. 32A07, 32H35, 32M05.

Key words and phrases. biholomorphic equivalence, Hua domain, automorphism group.

*Corresponding author. 
the explicit form of its automorphism group. There are other beautiful works about biholomorphic mapping and automorphism group on some kind of Hartogs domains, please refer to Ahn-Byun-Park [1] and the references therein for details.

In this paper, we will focus our attention on a well-known Hartogs domain called Hua domain. For an irreducible bounded symmetric domain $\Omega \subset \mathbb{C}^{n}$ in its Harish-Chandra realization, the Hua domain $H_{\Omega}(\mathbf{p})$ is defined by

$$
H_{\Omega}(\boldsymbol{p}):=\left\{\left(z, \omega_{(1)}, \ldots, \omega_{(m)}\right) \in \Omega \times \mathbb{C}^{n_{1}} \times \cdots \times \mathbb{C}^{n_{m}}: \sum_{j=1}^{m}\left\|\omega_{(j)}\right\|^{2 p_{j}}<N_{\Omega}(z, \bar{z})\right\},
$$

where $\boldsymbol{p}=\left(p_{1}, p_{2}, \ldots, p_{m}\right) \in \mathbb{R}_{+}^{m}$ and $N_{\Omega}(z, \bar{z})$ is a generic norm of $\Omega$ defined by

$$
N_{\Omega}(z, \xi):=(V(\Omega) K(z, \xi))^{-1 / \gamma},
$$

where $V(\Omega)$ is the total volume of $\Omega$ with respect to the Euclidean measure of $\mathbb{C}^{n}, K(z, \xi)$ is its Bergman kernel with respect to the Euclidean measure on $\mathbb{C}^{n}$, and $\gamma$ is the genus for the irreducible bounded symmetric domain $\Omega$. In general, one can check that the Hua domain is a nonhomogeneous bounded pesudoconvex domain without smooth boundary.

In particular, when $p_{1}=\cdots=p_{m}=1$, the Hua domain $H_{\Omega}(\boldsymbol{p})$ degenerates into Cartan-Hartogs domain. There are also many deep results on this domain. For studies of Cartan-Hartogs domain, the reader is referred to [1, 2,5, 9] and references therein.

Recently, Tu and Wang [16] studied the rigidity of proper holomorphic mappings between two equidimensional Hua domains and determined the explicit form for the biholomorphisms between two equidimensional Hua domains without checking $\Omega$ case by case. Their results are very impressive. Besides, Guo, Feng and Bi [11] also studied a rigidity of automorphism of Fock-Bargmann-Hartogs domains. Inspired by this, we will give an equivalent description of biholomorphic mapping of two equidimensional Hua domains.

We should note that if a holomorphic mapping keeping 1.2 invariant between two equidimensional Hua domains can be continuously extended to the boundary, it automatically becomes a proper holomorphic mapping between this two equidimensional Hua domains. Therefore by Tu and Wang's result (see [16. Theorem 1.3]), it is biholomorphic, provided that $\min \left\{n_{1+\delta}, \ldots, n_{m}, n_{1}+\cdots+n_{m}\right\} \geq 2$. However, it is still an open problem for the case where all the fibre's dimension equals one, namely the case for $n_{1}=\cdots=n_{m}=1$.

Hence, in this paper we will give an equivalent conditions for a holomorphic mapping between two equidimensional Hua domains with one dimension fibre to be biholomorphic. More precisely, we mainly focus our attention on a specialized Hua domain where we denote by $H_{\Omega}(p)$, which is defined by

$$
H_{\Omega}(p)=\left\{(z, \omega) \in \Omega \times \mathbb{C}^{m} \subset \mathbb{C}^{n} \times \mathbb{C}^{m}: \sum_{j=1}^{m}\left|\omega_{j}\right|^{2 p}<N_{\Omega}(z, \bar{z})\right\},
$$


where $\Omega$ is an irreducible bounded symmetric domain in $\mathbb{C}^{n}$ in its Harish-Chandra realization. Throughout this paper we can always assume that the domain $H_{\Omega}(p)$ is written in its standard form (this was named by Tu-Wang [16]), that is, when $\Omega$ is the unit ball in $\mathbb{C}^{n}$, then $p \neq 1$ or $\operatorname{rank}(\Omega) \geq 2$. It is not hard to see that every Hua domain can be written in its standard form by relabelling the coordinates. Furthermore, here and henceforth we will use the following function defined on $H_{\Omega}(p)$, namely

$$
\mathcal{L}_{\Omega}(z, \omega)=N_{\Omega}(z, \bar{z})^{-1} \sum_{j=1}^{m}\left|\omega_{j}\right|^{2 p}
$$

Then our main result can be stated as follows.

Theorem 1.1. Let $H_{\Omega_{1}}(p)$ and $H_{\Omega_{2}}(q)$ be two equidimensional Hua domains in their standard form, where $\Omega_{1}$ and $\Omega_{2}$ are two equidimensional irreducible bounded symmetric domains in the Harish-Chandra realization. Assume that $F$ is a holomorphic mapping between $H_{\Omega_{1}}(p)$ and $H_{\Omega_{2}}(q)$. Then $H_{\Omega_{1}}(p)$ and $H_{\Omega_{2}}(q)$ are holomorphic equivalent if and only if $F$ satisfies the following property

$$
\mathcal{L}_{\Omega_{2}}(F(z, \omega))=\mathcal{L}_{\Omega_{1}}(z, \omega)
$$

Combining with Theorem 1.1 in [16, we can easily get the main result in Feng [10]. Here we remark that our method is more simple than Feng's method.

Corollary 1.2. Let $H_{\Omega_{1}}(p)$ and $H_{\Omega_{2}}(q)$ be two equidimensional Hua domains in their standard form, where $\Omega_{1}$ and $\Omega_{2}$ are two equidimensional irreducible bounded symmetric domains. Assume that $F(z, \omega)$ is a holomorphic mapping between $H_{\Omega_{1}}(p)$ and $H_{\Omega_{2}}(q)$. If

$$
\mathcal{L}_{\Omega_{2}}(F(z, \omega))=\mathcal{L}_{\Omega_{1}}(z, \omega)
$$

then we get $p=q$ and there exists a $G \in \Gamma(H(q))$, where $\Gamma(H(q))$ consists of all the following mapping $\Phi$ :

$$
\Phi(z, \omega)=\left(\varphi(z), e^{i \theta_{1}} \omega_{1} \frac{\left(N_{\Omega_{2}}\left(z_{0}, \overline{z_{0}}\right)\right)^{1 /(2 q)}}{\left(N_{\Omega_{2}}\left(z, \overline{z_{0}}\right)\right)^{1 / q}}, \ldots, e^{i \theta_{m}} \omega_{m} \frac{\left(N_{\Omega_{2}}\left(z_{0}, \overline{z_{0}}\right)\right)^{1 /(2 q)}}{\left(N_{\Omega_{2}}\left(z, \overline{z_{0}}\right)\right)^{1 / q}}\right)
$$

in which $\varphi(z) \in \operatorname{Aut}\left(\Omega_{2}\right)$ and $z_{0}=\varphi^{-1}(0)$ such that

$$
G \circ F(z, \omega)=(z A, \omega)
$$

with $A$ being a complex linear isomorphism of $\mathbb{C}^{n}$ and $A\left(\Omega_{1}\right)=\Omega_{2}$.

Furthermore, by our result, it is also easy to obtain the rigidity for the automorphism of Hua domains. 
Corollary 1.3. Let $H_{\Omega}(p)$ be the Hua domain in its standard form, and $F(z, \omega)$ is holomorphic self-mapping of $H_{\Omega}(p)$. Then $F(z, \omega)$ is an automorphism of $H_{\Omega}(p)$ if and only if $F(z, \omega)$ keeps the function $\mathcal{L}_{\Omega}(z, \omega)$ invariant, that is, $\mathcal{L}_{\Omega}(F(z, \omega))=\mathcal{L}_{\Omega}(z, \omega)$.

In particular, when $p=1$ this is exactly an description for the automorphism of Cartan-Hartogs domains. This case can also illustrate that writing Hua domains in its standard form is essential.

This paper is organized as follows. In Section 2, we first recall some basic notations and facts on bounded symmetric domains, and establish a revised Cartan Theorem on a similar Hua domain. In Section 3 , we prove that the Jacobian matrix of $F(z, \omega)$ at the origin is non-degenerate. Then by using Cartan Theorem, we give the proof of our main results.

\section{Preliminaries}

At the beginning, let us briefly recall some basic facts on irreducible bounded symmetric domain and its generic norm.

Let $\Omega$ be an irreducible bounded symmetric domain in $\mathbb{C}^{n}$ with rank $r$ in its HarishChandra realization. Then there exists the Jordan triple product on $\mathbb{C}^{n}$ associated with the Bergman kernel of $\Omega$ and the space $\mathbb{C}^{n}$ endowed with triple product is a simple Hermitian positive Jordan triple system. Let $e_{1}, e_{2}, \ldots, e_{r} \in \mathbb{C}^{n}$ be a frame for $\mathbb{C}^{n}$. Then each $z \in \mathbb{C}^{n}$ has the spectral decomposition

$$
z=k(z) \cdot\left(\lambda_{1}(z) e_{1}+\lambda_{2}(z) e_{2}+\cdots+\lambda_{r}(z) e_{r}\right)
$$

where $k(z) \in \mathcal{K}, \lambda_{1} \geq \lambda_{2} \geq \cdots \geq \lambda_{r} \geq 0$, and $\mathcal{K}$ is the connected component of the identity in the Lie group of the (complex linear) automorphisms of $\Omega$ leaving the origin fixed. It is well-known that irreducible bounded symmetric domain can be regarded as the unit ball of the spectral norm, that is,

$$
\Omega=\left\{z \in \mathbb{C}^{n}:\||z|\|<1\right\}
$$

where $\||\cdot|||$ denotes the spectral norm of $z$ (see [8]).

Then generic norm $N_{\Omega}(z, \bar{z})$ can be expressed by

$$
N_{\Omega}\left(z_{1}, \bar{z}_{2}\right)=m\left(1, z_{1}, \bar{z}_{2}\right)
$$

where $m\left(t, z_{1}, \bar{z}_{2}\right)$ is the generic minimal polynomial of $\mathbb{C}^{n}$ (refer to 8 ). It follows that

$$
N_{\Omega}(z, \bar{z})=\prod_{j=1}^{r}\left(1-\lambda_{j}^{2}(z)\right) .
$$


By using logarithmic expansion, 2.1) implies

$$
\ln N_{\Omega}(z, \bar{z})=-\sum_{k=1}^{\infty} \frac{1}{k} \sum_{j=1}^{r} \lambda_{j}^{2 k}(z), \quad z \in \Omega .
$$

One can prove that (cf. 8 )

$$
\sum_{j=1}^{r} \lambda_{j}^{2 k}(z)=\sum_{|\alpha|=|\beta|=k} f_{\alpha \beta} z^{\alpha} \bar{z}^{\beta}
$$

where $\alpha=\left(\alpha_{1}, \alpha_{2}, \ldots, \alpha_{n}\right), \alpha_{i} \in \mathbb{N}, 1 \leq i \leq n,|\alpha|=\sum_{i=1}^{n} \alpha_{i}$ and $z^{\alpha}=\prod_{i=1}^{n} z_{i}^{\alpha_{i}}$.

Let $z=k \cdot\left(\lambda_{1}(z) e_{1}+\lambda_{2}(z) e_{2}+\cdots+\lambda_{r}(z) e_{r}\right)$ be the spectral decomposition of $z$. For given $t \geq 0$, the spectral decomposition of $t z$ is $t z=k \cdot\left(t \lambda_{1}(z) e_{1}+t \lambda_{2}(z) e_{2}+\cdots+t \lambda_{r}(z) e_{r}\right)$. By (2.1), we have

$$
N_{\Omega}(t z, \overline{t z})=\prod_{j=1}^{r}\left(1-t^{2} \lambda_{j}^{2}(z)\right)=1-t^{2} \sum_{j=1}^{r} \lambda_{j}^{2}(z)+\cdots .
$$

Moreover, one can see that (also see 8])

$$
\sum_{j=1}^{r} \lambda_{j}^{2}(z)=z C_{\Omega} \bar{z}^{t}
$$

where $C_{\Omega}$ is a positive definite Hermite matrix. For the proofs of above facts and details, please refer, e.g., to 8].

In the following, we give some properties of the generic norm of bounded symmetric domain.

Lemma 2.1. [16, Proposition 2.1] Let $\Omega$ be an irreducible bounded symmetric domain in $\mathbb{C}^{n}$ and let $N_{\Omega}(z, \bar{z})$ be the generic norm of $\Omega$. Then we have the results as follows:

(1) For any $z_{0} \in \Omega, N_{\Omega}\left(t z_{0}, \overline{t z_{0}}\right)(0 \leq t \leq 1)$ is a decreasing function of $t$.

(2) We have

$$
N_{\Omega}(z, 0)=1 \quad \text { and } \quad 0 \leq N_{\Omega}(z, \bar{z}) \leq 1, \quad z \in \Omega
$$

and $N_{\Omega}(z, \bar{z})=1$ if and only if $z=0$.

Lemma 2.2 (Cartan theorem). Let $D_{1}$ and $D_{2}$ be two bounded circular domains in $\mathbb{C}^{n}$ with $0 \in D_{i}(i=1,2)$. Suppose that $F: D_{1} \rightarrow D_{2}$ is a biholomorphic mapping and $F(0)=0$. Then $F$ is a linear transformation.

By Cartan theorem, we can easily get the following result.

Theorem 2.3. Let $F: N_{\Omega_{1}}^{-1}(z, \bar{z}) \sum_{j=1}^{m}\left|\omega_{j}\right|^{2 p}<\epsilon \rightarrow N_{\Omega_{2}}^{-1}(z, \bar{z}) \sum_{j=1}^{m}\left|\omega_{j}\right|^{2 q}<\epsilon^{\prime}$ be a biholomorphic mapping where $\epsilon$ and $\epsilon^{\prime}$ are small enough numbers. If $F(0)=0$, then $F$ is a linear transformation. 


\section{Proof of the main theorem}

Proof of Theorem 1.1. First, we know by [16] that $F(z, \omega)$ can be expressed by

$$
F(z, \omega)=\Phi \circ\left(z A, \omega_{\sigma(1)}, \ldots, \omega_{\sigma(m)}\right)
$$

where

$$
\Phi(z, \omega)=\left(\varphi(z), e^{i \theta_{1}} \omega_{1} \frac{\left(N_{\Omega_{2}}\left(z_{0}, \overline{z_{0}}\right)\right)^{1 /(2 q)}}{\left(N_{\Omega_{2}}\left(z, \overline{z_{0}}\right)\right)^{1 / q}}, \ldots, e^{i \theta_{m}} \omega_{m} \frac{\left(N_{\Omega_{2}}\left(z_{0}, \overline{z_{0}}\right)\right)^{1 /(2 q)}}{\left(N_{\Omega_{2}}\left(z, \overline{z_{0}}\right)\right)^{1 / q}}\right),
$$

$\varphi(z) \in \operatorname{Aut}\left(\Omega_{2}\right), z_{0}=\varphi^{-1}(0)$ and $p=q$. Since $A$ is a biholomorphic complex linear isomorphism of $\mathbb{C}^{n}$ with $A\left(\Omega_{1}\right)=\Omega_{2}$, it is easy to check that $\mathcal{L}_{\Omega_{2}}(T(z, \omega))=\mathcal{L}_{\Omega_{1}}(z, \omega)$, where $T(z, \omega)$ denotes the biholomorphic mapping

$$
T(z, \omega): H_{\Omega_{1}}(p) \rightarrow H_{\Omega_{2}}(q), \quad(z, \omega) \mapsto\left(z A, \omega_{\sigma(1)}, \ldots, \omega_{\sigma(m)}\right) .
$$

Next we will prove that $\mathcal{L}_{\Omega_{2}} \circ \Phi(z, \omega)=\mathcal{L}_{\Omega_{2}}(z, \omega)$.

Using the following equality

$$
\frac{N_{\Omega_{2}}(\varphi(z), \overline{\varphi(z)}) N_{\Omega_{2}}(\varphi(t), \overline{\varphi(t)})}{N_{\Omega_{2}}(\varphi(z), \overline{\varphi(t)})}=\frac{N_{\Omega_{2}}(z, \bar{z}) N_{\Omega_{2}}(t, \bar{t})}{\left|N_{\Omega_{2}}(z, \bar{t})\right|^{2}}
$$

and let $t=z_{0}$, where $z_{0}=\varphi^{-1}(0)$, we can get

$$
\frac{N_{\Omega_{2}}(\varphi(z), \overline{\varphi(z)}) N_{\Omega_{2}}(0, \overline{0})}{N_{\Omega_{2}}(\varphi(z), \overline{0})}=\frac{N_{\Omega_{2}}(z, \bar{z}) N_{\Omega_{2}}\left(z_{0}, \overline{z_{0}}\right)}{\left|N_{\Omega_{2}}\left(z, \overline{z_{0}}\right)\right|^{2}},
$$

which means

$$
N_{\Omega_{2}}(\varphi(z), \overline{\varphi(z)})=\frac{N_{\Omega_{2}}(z, \bar{z}) N_{\Omega_{2}}\left(z_{0}, \overline{z_{0}}\right)}{\left|N_{\Omega_{2}}\left(z, \overline{z_{0}}\right)\right|^{2}}
$$

It follows that

$$
\begin{aligned}
\mathcal{L}_{\Omega_{2}} \circ \Phi(z, \omega) & =\frac{\sum_{j=1}^{m}\left|e^{i \theta_{j}} \omega_{j} \frac{N_{\Omega_{2}}\left(z_{0}, \overline{z_{0}}\right)^{1 /(2 q)}}{N_{\Omega_{2}}\left(z, \overline{z_{0}}\right)^{1 / q}}\right|^{2 q}}{N_{\Omega_{2}}(\varphi(z), \overline{\varphi(z)})}=\frac{\sum_{j=1}^{m}\left|\omega_{j}\right|^{2 q} \frac{N_{\Omega_{2}}\left(z_{0}, \overline{z_{0}}\right)}{\left|N_{\Omega_{2}}\left(z, \overline{z_{0}}\right)\right|^{2}}}{\frac{N_{\Omega_{2}}(z, \bar{z}) N_{\Omega_{2}}\left(z_{0}, \overline{z_{0}}\right)}{\left|N_{\Omega_{2}}\left(z, \overline{z_{0}}\right)\right|^{2}}} \\
& =\frac{\sum_{j=1}^{m}\left|\omega_{j}\right|^{2 q}}{N_{\Omega_{2}}(z, \bar{z})}=\mathcal{L}_{\Omega_{2}}(z, \omega) .
\end{aligned}
$$

Thus $\Phi(z, \omega)$ keeps $\mathcal{L}_{\Omega_{2}}(z, \omega)$ invariant. In summary, we can learn that $\mathcal{L}_{\Omega_{2}} \circ F(z, \omega)=$ $\mathcal{L}_{\Omega_{1}}(z, \omega)$.

In the following, we will prove that the converse also holds, which will be divided into three steps.

Step 1. Let $F(z, \omega)=\left(F_{1}(z, \omega), F_{2}(z, \omega)\right)=\left(F_{11}(z, \omega), \ldots, F_{1 n}(z, \omega), F_{21}(z, \omega), \ldots\right.$, $\left.F_{2 m}(z, \omega)\right)$. By the fact that $\mathcal{L}_{\Omega_{2}}(F(z, 0))=\mathcal{L}_{\Omega_{1}}(z, 0)$, we obtain

$$
\frac{\sum_{j=1}^{m}\left|F_{2 j}(z, 0)\right|^{2 q}}{N_{\Omega_{2}}\left(F_{1}(z, 0), \overline{F_{1}(z, 0)}\right)}=0 \text {. }
$$


Thus

$$
\sum_{j=1}^{m}\left|F_{2 j}(z, 0)\right|^{2 q}=0 .
$$

Then we can see that $F_{2}(z, 0)=0$. It follows $F(0,0)=(u, 0) \in \Omega_{2} \times\{0\}$. Consider a biholomorphic self-mapping $G$ of $H_{\Omega_{2}}(q)$ which is defined by

$$
G(z, \omega)=\left(\chi(z), \omega \frac{N_{\Omega_{2}}(u, \bar{u})^{1 /(2 q)}}{N_{\Omega_{2}}(z, \bar{u})^{1 / q}}\right),
$$

where $\chi(z) \in \operatorname{Aut}\left(\Omega_{2}\right)$ with $\chi(0)=u$. Then $G(z, \omega)$ maps $(0,0)$ to $(u, 0)$. We notice that $G(z, \omega)$ keeps the function $\mathcal{L}_{\Omega_{2}}$ on $H_{\Omega_{2}}(q)$ invariant. Let $H=G^{-1} \circ F$, then $H(0,0)=(0,0)$ and $\mathcal{L}_{\Omega_{2}} \circ H=\mathcal{L}_{\Omega_{1}}$. Hence we have

$$
H(z, \omega)=\left(H_{1}(z, \omega), H_{2}(z, \omega)\right)=(z, w) T+\text { higher order terms }
$$

where

$$
T=\left(\begin{array}{ll}
A & B \\
V & U
\end{array}\right) .
$$

Since $H_{2}(z, 0)=0$, we have $B=0$. Now assume that $H(0, \omega)=\left(h_{1}(\omega), h_{2}(\omega)\right)$, in which

$$
h_{1}(\omega)=\omega V+\sum_{i \geq 2} f_{i}(\omega), \quad h_{2}(\omega)=\omega U+\sum_{i \geq 2} g_{i}(\omega)
$$

where $f_{i}(\omega)$ and $g_{i}(\omega)$ are homogeneous polynomials of degree $i(i \geq 2)$. For any $(0, \omega) \in$ $H_{\Omega_{1}}(p)$, we obtain

$$
\sum_{j=1}^{m}\left|\omega_{j}\right|^{2 p}<1
$$

For any $t \in[0,1]$, we can see $(0, t \omega) \in H_{\Omega_{1}}(p)$. By $\mathcal{L}_{\Omega_{2}} \circ H(0, t \omega)=\mathcal{L}_{\Omega_{1}}(0, t \omega)$, we have

$$
\frac{\sum_{j=1}^{m}\left|\left(h_{2}(t \omega)\right)_{j}\right|^{2 q}}{N_{\Omega_{2}}\left(h_{1}(t \omega), \overline{h_{1}(t \omega)}\right)}=\sum_{j=1}^{m}\left|t \omega_{j}\right|^{2 p} .
$$

Substituting $h_{1}(t \omega)$ and $h_{2}(t \omega)$ into (3.1), we obtain

$$
\frac{\sum_{j=1}^{m}\left|\left(t \omega U+\sum_{i \geq 2} g_{i}(t \omega)\right)_{j}\right|^{2 q}}{\sum_{j=1}^{m}\left|t \omega_{j}\right|^{2 p}}=N_{\Omega_{2}}\left(t \omega V+\sum_{i \geq 2} f_{i}(t \omega), \overline{t \omega V+\sum_{i \geq 2} f_{i}(t \omega)}\right) .
$$

This leads to

$$
\frac{t^{2 q} \sum_{j=1}^{m}\left|\left(\omega U+\sum_{i \geq 2} t^{i-1} g_{i}(\omega)\right)_{j}\right|^{2 q}}{t^{2 p} \sum_{j=1}^{m}\left|\omega_{j}\right|^{2 p}}=N_{\Omega_{2}}\left(t \omega V+\sum_{i \geq 2} f_{i}(t \omega), \overline{t \omega V+\sum_{i \geq 2} f_{i}(t \omega)}\right) .
$$


Taking $t \rightarrow 0^{+}$, we get $q=p$. Otherwise, the limit on the left-hand side of this equation doesn't exist or is 0 . Hence we will write $q$ as $p$ in the following proof.

Then we obtain

$$
\sum_{j=1}^{m}\left|(\omega U)_{j}\right|^{2 p}=\sum_{j=1}^{m}\left|\omega_{j}\right|^{2 p}
$$

It follows that $\omega \rightarrow \omega U$ is a biholomorphic self-maps of complex ellipsoid $\left\{\omega \in \mathbb{C}^{m}\right.$ : $\left.\sum_{j=1}^{m}|\omega|^{2 p}<1\right\}$. Thus the mapping $L(z, w)$ defined by

$$
L(z, \omega)=(z, \omega U), \quad(z, \omega) \in \mathbb{C}^{n} \times \mathbb{C}^{m}
$$

induces a holomorphic automorphism of $H_{\Omega_{2}}(q)$. Thus by [16], we can see that

$$
\omega U=\left(\omega_{\sigma(1)}, \ldots, \omega_{\sigma(m)}\right)\left(\begin{array}{ccc}
e^{i \theta_{1}} & & \\
& \ddots & \\
& & e^{i \theta_{m}}
\end{array}\right),
$$

where $\sigma$ denotes a permutation of $\{1, \ldots, m\}$. Therefore, taking the composite mapping $H \circ L^{-1}$ if necessary, we can assume that

$$
h_{2}(\omega)=\omega+\sum_{i \geq 2} g_{i}(\omega)
$$

Moreover, applying the Cartan Lemma to $h_{2}(\omega)$, we must have $h_{2}(\omega)=\omega$, namely $U=$ $I_{m \times m}$. Hence we have $H(0, \omega)=\left(h_{1}(\omega), \omega\right)$. Since $\mathcal{L}_{2} \circ H(0, \omega)=\mathcal{L}_{1}(0, \omega)$, it follows that

$$
\frac{\sum_{j=1}^{m}\left|\omega_{j}\right|^{2 p}}{N_{\Omega_{2}}\left(h_{1}(\omega), \overline{h_{1}(\omega)}\right)}=\sum_{j=1}^{m}\left|\omega_{j}\right|^{2 p}
$$

It yields that $N_{\Omega_{2}}\left(h_{1}(\omega), \overline{h_{1}(\omega)}\right)=1$ which implies $h_{1}(\omega)=0$ by Lemma 2.1 , and $V=0$. Therefore we have

$$
H_{1}(z, \omega)=z A+\sum_{i \geq 2} P_{i}(z, \omega), \quad H_{2}(z, \omega)=\omega+\sum_{i \geq 2} Q_{i}(z, \omega)
$$

where $P_{j}$ and $Q_{j}$ are homogeneous polynomials of degree $j$.

Step 2. In this step, we will show that the matrix $A$ is an invertible matrix. Let $(z, \omega) \in H_{\Omega_{1}}(p)$. It is not hard to see that

$$
(t z, t \omega) \in H_{\Omega_{1}}(p), \quad \forall t \in[0,1]
$$

by Lemma 2.1. By $\mathcal{L}_{\Omega_{2}} \circ H(t z, t \omega)=\mathcal{L}_{\Omega_{2}}(t z, t \omega)$, we get

$$
\frac{\sum_{j=1}^{m}\left|\left(H_{2}(t z, t \omega)\right)_{j}\right|^{2 p}}{\sum_{j=1}^{m}\left|t \omega_{j}\right|^{2 p}}=\frac{N_{\Omega_{2}}\left(H_{1}(t z, t \omega), \overline{H_{1}(t z, t \omega)}\right)}{N_{\Omega_{1}}(t z, t \bar{z})} .
$$


Substituting $H_{1}(t z, t \omega)$ and $H_{2}(t z, t \omega)$ into $(3.3)$, we obtain

$$
\frac{\sum_{j=1}^{m}\left|\left(t \omega+\sum_{i \geq 2} Q_{i}(t z, t \omega)\right)_{j}\right|^{2 p}}{\sum_{j=1}^{m}\left|t \omega_{j}\right|^{2 p}}=\frac{N_{\Omega_{2}}\left(t z A+\sum_{i \geq 2} P_{i}(t z, t \omega), \overline{t z A+\sum_{i \geq 2} P_{i}(t z, t \omega)}\right)}{N_{\Omega_{1}}(t z, t \bar{z})} .
$$

Therefore we get

$$
\begin{aligned}
& \frac{\sum_{j=1}^{m}\left|\omega_{j}+\left(\sum_{i \geq 2} t^{i-1} Q_{i}(z, \omega)\right)_{j}\right|^{2 p}}{\sum_{j=1}^{m}\left|\omega_{j}\right|^{2 p}} \\
= & \frac{N_{\Omega_{2}}\left(t z A+\sum_{i \geq 2} P_{i}(t z, t \omega), \overline{t z A+\sum_{i \geq 2} P_{i}(t z, t \omega)}\right)}{N_{\Omega_{1}}(t z, t \bar{z})} .
\end{aligned}
$$

For the sake of simplicity, we write $R_{j}(t, z, \omega)=\left(\sum_{i \geq 2} t^{i-1} Q_{i}(z, \omega)\right)_{j}(1 \leq j \leq m)$. A straightforward computation yields that $\left(\omega_{j} \neq 0,1 \leq j \leq m\right)$

$$
\frac{\sum_{j=1}^{m}\left|\omega_{j}+R_{j}(t, z, \omega)\right|^{2 p}}{\sum_{j=1}^{m}\left|\omega_{j}\right|^{2 p}}=1+\frac{\sum_{j=1}^{m}\left|\omega_{j}\right|^{2 p}\left(p u_{j}+\frac{p(p-1)}{2} u_{j}^{2}+o\left(t^{2}\right)\right)}{\sum_{j=1}^{m}\left|\omega_{j}\right|^{2 p}},
$$

where $u_{j}=T_{j}(t, z, \omega) /\left|\omega_{j}\right|^{2}$ and $T_{j}(t, z, \omega)$ is defined by

$$
\begin{aligned}
T_{j}(t, z, \omega) & :=2 \operatorname{Re}\left(\bar{\omega}_{j} R_{j}(t, z, \omega)\right)+\left|R_{j}(t, z, \omega)\right|^{2} \\
& =t\left(2 \operatorname{Re}\left(\bar{\omega}_{j} Q_{2, j}(z, \omega)\right)\right)+t^{2}\left(2 \operatorname{Re}\left(\bar{\omega}_{j} Q_{3, j}(z, \omega)\right)+\left|Q_{2, j}(z, \omega)\right|^{2}\right) .
\end{aligned}
$$

Combining (3.4) and (3.5), we can learn that

$$
\begin{aligned}
& \ln \left(1+\frac{\sum_{j=1}^{m}\left|\omega_{j}\right|^{2 p}\left(p u_{j}+\frac{p(p-1)}{2} u_{j}^{2}+o\left(t^{2}\right)\right)}{\sum_{j=1}^{m}\left|\omega_{j}\right|^{2 p}}\right) \\
= & \ln \left(\frac{N_{\Omega_{2}}\left(t z A+\sum_{i \geq 2} P_{i}(t z, t \omega), \overline{t z A+\sum_{i \geq 2} P_{i}(t z, t \omega)}\right)}{N_{\Omega_{1}}(t z, t \bar{z})}\right) .
\end{aligned}
$$

By 2.2), we have

$$
\begin{aligned}
& \ln N_{\Omega_{2}}\left(t z A+\sum_{i \geq 2} P_{i}(t z, t \omega) \overline{t z A+\sum_{i \geq 2} P_{i}(t z, t \omega)}\right) \\
= & -t^{2} \sum_{l=1}^{r_{2}} \Lambda_{l}^{2}\left(z A+\sum_{i \geq 2} t^{i-1} P_{i}(z, \omega)\right)+o\left(t^{2}\right),
\end{aligned}
$$

and

$$
\ln N_{\Omega_{1}}(t z, t \bar{z})=-t^{2} \sum_{l=1}^{r_{1}} \lambda_{l}^{2}(z)+o\left(t^{2}\right)
$$


Substituting (3.7) and (3.8) into (3.6), and then taking $t \rightarrow 0^{+}$by dividing both sides of (3.6) by $t^{2}$, one can get

$$
\sum_{j=1}^{m} p\left|\omega_{j}\right|^{2(p-1)}\left(2 \operatorname{Re}\left(\bar{\omega}_{j} Q_{2, j}(z, \omega)\right)\right) \equiv 0
$$

Since $Q_{2, j}(z, \omega)$ are homogeneous polynomial functions of degree 2 , hence it is easy to get

$$
Q_{2, j}(z, \omega) \equiv 0, \quad 1 \leq j \leq m
$$

On the other hand, dividing both sides of (3.6) by $t^{2}$ and combining with above equation, we can see that

$$
\sum_{j=1}^{m} p\left|\omega_{j}\right|^{2(p-1)}\left(2 \operatorname{Re}\left(\bar{\omega}_{j} Q_{3, j}(z, \omega)\right)\right)+\sum_{j=1}^{m}\left|\omega_{j}\right|^{2 p} S_{2}(z, \bar{z}) \equiv 0
$$

where

$$
S_{2}(z, \bar{z})=\sum_{l=1}^{r_{2}} \Lambda_{l}^{2}(z A)-\sum_{l=1}^{r_{1}} \lambda_{l}^{2}(z)
$$

We also note that $Q_{3, j}(z, \omega)$ and $S_{2}(z, \bar{z})$ are homogeneous polynomial functions of degree 3 and degree 2 , respectively. Similarly we have $S_{2}(z, \bar{z})=0$. This implies that

$$
\sum_{l=1}^{r_{2}} \Lambda_{l}^{2}(z A)=\sum_{l=1}^{r_{1}} \lambda_{l}^{2}(z)
$$

By (2.3), we can learn that

$$
z A C_{\Omega_{2}} \bar{A}^{t} \bar{z}^{t}=z C_{\Omega_{1}} \bar{z}^{t}
$$

Thus $A$ is an invertible matrix.

Step 3. Since $A$ is invertible, it means that $H(z, \omega)$ is biholomorphic at a neighborhood $U$ of the origin. Furthermore, we can choose a small enough $\epsilon$ such that

$$
\widetilde{H}:=\left.H\right|_{N_{\Omega_{1}}^{-1} \sum_{j=1}^{m}\left|\omega_{j}\right|^{2 p}<\epsilon}: N_{\Omega_{1}}^{-1}(z, \bar{z}) \sum_{j=1}^{m}\left|\omega_{j}\right|^{2 p}<\epsilon \subset U \rightarrow N_{\Omega_{2}}^{-1}(z, \bar{z}) \sum_{j=1}^{m}\left|\omega_{j}\right|^{2 p}<\epsilon
$$

is biholomorphic by the fact that $\mathcal{L}_{\Omega_{2}} \circ H(z, \omega)=\mathcal{L}_{\Omega_{1}}(z, \omega)$. Therefore we have $\widetilde{H}(z, \omega)$ is a linear mapping by Theorem 2.3. This implies that $\widetilde{H}(z, \omega)=(z A, \omega)$ by 3.2 .

Obviously, the mapping $\widetilde{H}(z, \omega)$ can be holomorhically extended to $\overline{H_{\Omega_{1}}(p)}$. Therefore by the identify principle theorem, we can conclude that

$$
H(z, \omega)=(z A, \omega), \quad(z, \omega) \in H_{\Omega_{1}}(p)
$$

Therefore by Step 1 we can get that $F(z, \omega)$ is biholomorphic. The proof is finished. 
Proof of Corollary 1.2. By Theorem 1.1, we know that $F$ is biholomorphic. Then by Theorem 1.1 in [16], there exist an automorphism $G \in \Gamma\left(H_{\Omega}(p)\right)$ and a permutation $\sigma \in S_{m}$ with $p_{\sigma(i)}=q_{i}$ such that

$$
G \circ F(z, \omega)=(z A, \omega)
$$

where $z \in \Omega_{1} \mapsto z A \in \Omega_{2}$ is a biholomorphic mapping of $\Omega_{1}$ onto $\Omega_{2}$. Thus we have $p=q$. The proof is completed.

\section{Acknowledgments}

We sincerely thank the referee who read the paper carefully and gave many useful suggestions. The authors are also indebted to Prof. Z. M. Feng for many helpful discussions. This work was partly supported by the National Natural Science Foundation of China (No. 11901327) and Natural Science Foundation of Shandong Province (No. ZR2018BA015).

\section{References}

[1] H. Ahn, J. Byun and J.-D. Park, Automorphisms of the Hartogs type domains over classical symmetric domains, Internat. J. Math. 23 (2012), no. 9, 1250098, 11 pp.

[2] E. Bi, Z. Feng, G. Su and Z. Tu, Rawnsley's $\varepsilon$-function on some Hartogs type domains over bounded symmetric domains and its applications, J. Geom. Phys. 135 (2019), 187-203.

[3] E. Bi and G. Su, Balanced metrics and Berezin quantization on Hartogs triangles, Annali di Matematica Pura ed Applicata, 2020.

[4] E. Bi, G. Su and Z. Tu, The Kobayashi pseudometric for the Fock-Bargmann-Hartogs domain and its application, J. Geom. Anal. 30 (2020), no. 1, 86-106.

[5] E. Bi and Z. Tu, Remarks on the canonical metrics on the Cartan-Hartogs domains, C. R. Math. Acad. Sci. Paris 355 (2017), no. 7, 760-768.

[6] _ Rigidity of proper holomorphic mappings between generalized FockBargmann-Hartogs domains, Pacific J. Math. 297 (2018), no. 2, 277-297.

[7] G. Dini and A. Selvaggi Primicerio, Localization principle of automorphisms on generalized pseudoellipsoids, J. Geom. Anal. 7 (1997), no. 4, 575-584. 
[8] J. Faraut, S. Kaneyuki, A. Korányi, Q.-k. Lu and G. Roos, Analysis and Geometry on Complex Homogeneous Domains, Progress in Mathematics 185, Birkhäuser Boston, Boston, MA, 2000.

[9] Z. Feng, Hilbert spaces of holomorphic functions on generalized Cartan-Hartogs domains, Complex Var. Elliptic Equ. 58 (2013), no. 3, 431-450.

[10] _ A characterization of the biholomorphisms between equidimensional CartanHartogs domains, J. of Math. (PRC) 35 (2015), no. 4, 841-854.

[11] T. Guo, Z. Feng and E. Bi, Rigidity of the holomorphic automorphism of the generalized Fock-Bargmann-Hartogs domains, accepted in Czechoslovak Math. J., 14 pp. https://doi.org/10.21136/CMJ.2020.0364-19

[12] A. Kodama, On the holomorphic automorphism group of a generalized complex ellipsoid, Complex Var. Elliptic Equ. 59 (2014), no. 9, 1342-1349.

[13] A. Kodama, S. G. Krantz and D. Ma, A characterization of generalized complex ellipsoids in $\mathbf{C}^{n}$ and related results, Indiana Univ. Math. J. 41 (1992), no. 1, 173195.

[14] A. Loi and M. Zedda, Balanced metrics on Cartan and Cartan-Hartogs domains, Math. Z. 270 (2012), no. 3-4, 1077-1087.

[15] I. Naruki, The holomorphic equivalence problem for a class of Reinhardt domains, Publ. Res. Inst. Math. Sci. A. 4 (1968), 527-543.

[16] Z. Tu and L. Wang, Rigidity of proper holomorphic mappings between equidimensional Hua domains, Math. Ann. 363 (2015), no. 1-2, 1-34.

Ting Guo

School of Mathematics and Statistics, Qingdao University, Qingdao, Shandong 266071, China

E-mail address: guoting@qdu.edu.cn

Huan Yang

School of Mathematics and Physics, Qingdao University of Science and Technology,

Qingdao, Shandong 266061, China

E-mail address: huanyang@whu.edu.cn 